\title{
Impurity Energy Level Within The Haldane Gap
}

\author{
Wei Wang, ${ }^{1}$ Shaojin Qin, ${ }^{2}$ Zhong-Yi Lu, ${ }^{3} \mathrm{Lu} \mathrm{Yu},{ }^{2,4}$ and Zhaobin $\mathrm{Su}^{2}$ \\ 1 Physics Department of Peking University, Beijing 100871, China \\ ${ }^{2}$ Institute of Theoretical Physics, Academia Sinica, Beijing 100080, China \\ ${ }^{3}$ International School for Advanced Studies, 34013 Trieste, Italy \\ ${ }^{4}$ International Centre for Theoretical Physics, PO. Box 586, 34100 Trieste, Italy
}

\begin{abstract}
An impurity bond $J^{\prime}$ in a periodic 1D antiferromagnetic, spin 1 chain with exchange $J$ is considered. Using the numerical density matrix renormalization group method, we find an impurity energy level in the Haldane gap, corresponding to a bound state near the impurity bond. When $J^{\prime}<J$ the level changes gradually from the edge of the Haldane gap to the ground state energy as the deviation dev $=\left(J-J^{\prime}\right) / J$ changes from 0 to 1 . It seems that there is no threshold. Yet, there is a threshold when $J^{\prime}>J$. The impurity level appears only when the deviation $\operatorname{dev}=\left(J^{\prime}-J\right) / J^{\prime}$ is greater than $B_{c}$, which is near 0.3 in our calculation.
\end{abstract}

PACS numbers:75.10Jm,76.50+g,75.50.Ee 


\section{INTRODUCTION}

The Heisenberg model of $1 D$ antiferromagnetic(AF) chains is :

$$
H=J \sum_{i} \mathbf{S}_{i} \cdot \mathbf{S}_{i+1}
$$

with $J>0$, and $\mathbf{S}_{i}$ is a quantum spin. Up till now, the exact solution of this Hamiltonian is available only when $s=1 / 2$. The solution is obtained by the Bethe ansatz [1], which shows there is no gap in the energy spectrum. Then, what is the situation when $s=1$ or other integer? Some years ago, Haldane made his famous conjecture [2] that $1 D$ AF chains of integer spin have a gap, while those of half integer spin have a gapless spectrum. Since then, a lot of work has been done. In particular, a Valence-Bond-Solid (VBS) picture was proposed by Affleck, Kennedy, Lieb, and Tasaki(AKLT) [3, [4 to interprete the ground state of the integer spin AF chains. The proposed picture agrees quite well with both experimental [5] and numerical [6] studies.

An important issue of integer spin AF chains is the doping effect which involves some fundamental many-body quantum problems [0]]. DiTusa et al [8] have done doping experiments with $\mathrm{Zn}$ or $\mathrm{Ca}$ in $\mathrm{Y}_{2} \mathrm{BaNiO}_{5}$, which contains $1 \mathrm{D}$ AF chains of $\mathrm{Ni}-(\mathrm{O})-\mathrm{Ni}-(\mathrm{O})-\cdots$. In the $Z n$ doping case, the non-magnetic $Z n^{2+}$ ion substitutes $N i^{2+}$ to sever the AF chain giving rise to finite length effect. On the other hand, in the $\mathrm{Ca}$ doping case $\left(\mathrm{Ca}^{2+}\right.$ substituting the off-chain atom $\mathrm{Y}^{3+}$ ), holes are introduced in the oxygen orbitals along the $\mathrm{Ni}-\mathrm{O}$ chain, modifying the superexchange interaction and producing an impurity state inside the Haldane gap, as seen by neutron scattering experiments. The latter case can be represented by changing $J$ to $J^{\prime}$ at the impurity bond. We focus on this case in this Brief Report. Thus we write down our Hamiltonian as:

$$
H=J \sum_{i} \mathbf{S}_{i} \cdot \mathbf{S}_{i+1}+\left(J^{\prime}-J\right) \mathbf{S}_{0} \cdot \mathbf{S}_{1},
$$

where the impurity bond is put between sites 0 and 1 .

Recently a calculation of the dynamic structure factor based on the Schwinger-boson approach was performed for the bond-doping case []], which first indicated the existence of 
a threshold for the bond-coupling deviation. According to this calculation, a bound state is induced by the impurity only if the deviation exceeds this threshold, and the impurity level is located in the middle of the Haldane gap, almost independent of bond-coupling when temperature is much lower than the Haldane gap.

The recently developed numerical method of density matrix renormalization group (DMRG) [9, 10] has achieved a great success in calculating the low energy spectrum of 1D Heisenberg AF chains [11], so it's interesting and natural to use this method to study the doping effect on the Haldane gap systems. Such a calculation was carried out by Sorenson and Affleck (SA) [12]. They reported the existence of the threshold, but the impurity energy level they obtained changes gradually as the strength of impurity bond changes. The existence of the threshold is in agreement with the Schwinger boson calculation [0], while the gradual change of the impurity level is in disagreemnet with the latter. However, these authors have used the open boundary conditions and the way they added new sites may affect the bound state itself (See the discussion in the next Section). To clarify this issue we have decided to devise a more elaborate scheme to carry out the DMRG calculations.

\section{DMRG METHOD}

The DMRG method has been developed by S.R. White et al [9.10] in recent years. Generally, the standard pattern looks like this:

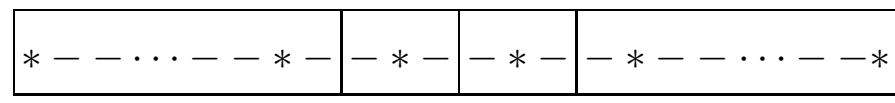

During each iteration, the chain (superblock) is divided into 4 blocks, where the leftmost and the right-most (which can be named as old blocks) are the same for the symmetry consideration. The two middle blocks contain only two sites. After each run, the left and right halves of the superblock are optimized to form new blocks which are used for the next iteration. This is, however, not good in the presence of an impurity bond. At first glance it seems right to put the impurity bond in the middle of the chain (this is what SA have 
done [12]), but this configuration may introduce some artificial effects. For example, the two middle sites which were optimized with the impurity bond between them, are used in later calculations without impurity being close to them. When the chain is very long, the impurity bond effect should go to zero during iterations (since there is only one impurity bond), but using this pattern, the effect cannot become small as iterations go on.

To calculate the impurity level of the periodic chain, we do our numerical work using the following pattern:

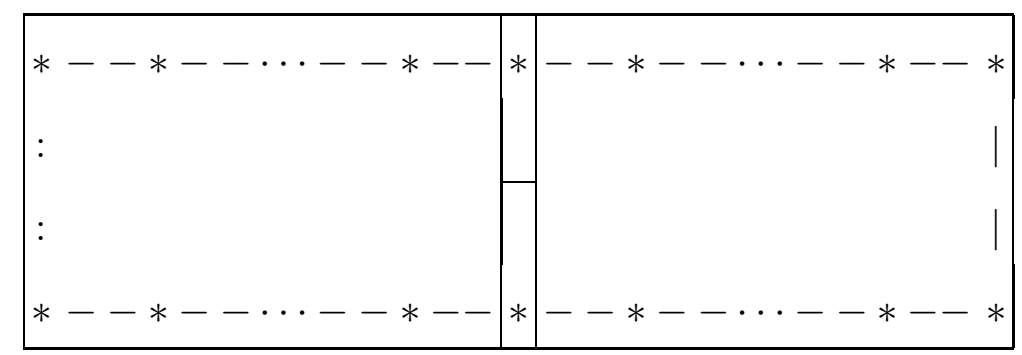

The impurity bond is placed at the left-hand side, and we add two sites each time with one each at or near the center of the upper and lower parts of the above periodic chain. Using this pattern, one can avoid the difficulties mentioned above. We preserve $m=100$ states in each run and discard the rest. Since $S_{z}$ is a good quantum number, we use it to reduce our Hamiltonian matrix.

Shank transformation has been demonstrated as a powerful extrapolation method to get Haldane gap precisely [13]. We use it to extrapolate our results to the infinite length limit. To verify the credibility of this method, we also use Aitkens' transformation [14] to extrapolate our finite chain data. They both give very similar values (usually the difference is less than $10^{-3}$ ). Compared with the previous conclusion in special cases, our results show reasonable precision. So we believe that our value is correct at least up to $10^{-2}$.

\section{NUMERICAL RESULT}

We use VBS picture [3,4] to give some intuitive explanation of our calculation results, although this picture is not quite precise. According to this picture, when considering the 
low excitation energy spectrum, the spin 1 of each site can be regarded as two spin $1 / 2^{\prime} s$ combined together to form a triplet $S=1$ state, yet the two $1 / 2$ spins of the nearest sites form a Valence-Bond, or, a singlet state. Therefore, the ground state of an open chain can be labelled by the sum of the two edge sites $1 / 2$ spins. The ground state of the $1 \mathrm{D}$ AF chain is four-fold degenerate, with one state of $\mathbf{S}_{\text {total }}=0$, where the two $1 / 2$ spins of each edge form a singlet, and three states of $\mathbf{S}_{\text {total }}=1$, where the two $1 / 2$ spins form a triplet. The first excited states are the quintlet states with $\mathbf{S}_{\text {total }}=2$, which lie above the ground state by the famous Haldane gap. Above them, the continuous spectrum starts. For a periodic chain, VBS picture also holds, but the triplet state rises up to the edge of the continuous energy spectrum, leaving the ground state nondegenerate.

The impurity state of the periodic chain, is just the $\mathbf{S}_{\text {total }}=1$ triplet state. When $J^{\prime} / J=0$, the periodic chain becomes an open chain, and this triplet will merge with the $\mathbf{S}_{\text {total }}=0$ ground state in the infinite chain length limit. Thus the impurity energy level is at 0 . On the contrary, when $J^{\prime} / J=1$, the periodic chain recovers, so the "impurity" level will rise up to merge with the Haldane gap.

Because of the $S O(3)$ symmetry of the Heisenberg Hamiltonian, the $\mathbf{S}_{\text {total }}=1$ triplets are degenerate, so we choose the state $S_{z}=1$ as a representative of them. Also we set the zero of the energy spectrum as the ground state energy.

First we consider the case $0<J^{\prime}<J$, which means the super- exchange is smaller due to doping, yet it does not change the AF nature. The calculated result is presented in Figure 1, from which we see that the impurity level increases linearly near dev $=1\left(\operatorname{dev}=\left(J-J^{\prime}\right) / J\right)$, which is in accordance with SA's perturbation argument [12]. However, compared with SA's work [12], our numerical result favors the conclusion that there is no threshold. As seen from the figure, the impurity energy appears as soon as $d e v \neq 0$, and shows a quadratic behavior near $d e v=0$. Kaburagi et al [15] has investigated the impurity bond effect in terms of domain wall exitations. Their variational results show the linear behavior near $d e v=0$ and quadratic behavior near dev $=1$. They also perform diagonalizations by Lanczos method for $N=12$ and 13 chains to support the variational results. 
When $J^{\prime}>J$, the result is shown in Figure 2, where the impurity level vs dev $=$ $\left(J^{\prime}-J\right) / J^{\prime}$ is plotted. This time, a threshold $B_{c}$ appears near 0.3, i.e., only when $\operatorname{dev}>B_{c}$ an impurity localized state will appear. Again the level rises linearly near $\operatorname{dev}=1$, in agreement with the results of SA [12], but with a different $B_{c}$ (they gave $B_{c}=0.5$ ). The case when $J^{\prime} / J \rightarrow \infty($ dev $\rightarrow 1)$ can be understood easily as follows: The two spins of the impurity bond form a singlet state because of the strong coupling, breaking the periodic chain of length L to make an open chain of length L-2. So, as following from our calculation, the difference between the impurity level and the ground state will go to zero in this limit. According to the finit chain results, the impurity level when $0<\operatorname{dev}<B_{c}$ is greater than the level when $d e v=0$, and the difference between them become smaller when the chain length increases,so we believe there is a threshold when the length goes to infinity, instead of the exponential or other behavior of the energy near dev $=0$.

Moreover, to ensure that the impurity level means a local state near the impurity bond, we plot $<S_{z i}>$ of the periodic chain in Figure $3\left(J^{\prime}<J\right)$. When $J^{\prime} / J=0(\operatorname{dev}=1)$, the "boundary $1 / 2$ spin" of open chain appears, just as the VBS picture assumes [3]. When dev goes from 1 to 0 , the state becomes more and more delocalized. Figure 4 shows the case of $J^{\prime}>J$, where the two sites at the middle form a singlet state, while the sites near them show the "boundary $1 / 2$ spin", too, when dev $\rightarrow 1$.

Figure 5 and 6 give the Shank transformation results of $|d E / d D e v|$. There is obviously no abrupt change.

\section{CONCLUSION}

Using an elaborate numerical DMRG method, we have investigated the bond doping effect of $1 \mathrm{D}$ AF chain. A threshold $B_{c}$ exists only when $J^{\prime}>J$. When $J^{\prime}<J$ or $J^{\prime}>J$ but $\operatorname{dev}<B_{c}$, no impurity state is induced. When $\operatorname{dev}>B_{c}$ and $J^{\prime}>J$, the impurity level within the Haldane gap corresponds to a local state near the impurity bond, and the level changes gradually when dev runs from $B_{c}$ to 1 . The discrepancy of the present study 
with the Schwinger boson calculation [7] is not fully understood at present. One possiblity is that the Schwinger boson representation introduces an additional symmetry (which sets the impurity level at the middle of the Haldane gap), not inherent to the original quantum spin systems.

Our calculation is supported by LSEC, CCAST and Laboratory of Computational Physics,IAPCM. We appreciate their assistance. After completion of our calculations we received a preprint by Xiaoqun Wang and S. Mallwitz, which reports essentially the same results. We thank Dr. Wang for sending us the preprint. 


\section{REFERENCES}

[1] H.A. Bethe, Z. Phys. 71, 205 (1931).

[2] F.D. Haldane, Phys. Lett. 93A, 464 (1983); Phys. Rev. Lett. 50,1153 (1983).

[3] I. Affleck,T. Kennedy, E.H. Lieb and H.Tasaki, Phys. Rev. Lett. 59, 799 (1987).

[4] I. Affleck, T. Kennedy, E.H. Lieb and H. Tasaki, Commun. Math. Phys.147, 431 (1992).

[5] See,e.g., J.P.Renard et al, Europhys. Lett. 3,945 (1987); W. Lu,et al Phys. Rev. Lett. 67, 3538 (1988); L.C. Brunel et al Phys. Rev. Lett. , 69,1699 (1992).

[6] See, e.g. O. Golinelli et al, Phys. Rev. B 50,3037(1994); J.B. Parkinson and J.C. Bonner, Phys. Rev. B 32,4703(1985); S. Liang, Phys. Rev. Lett. 64,1597 (1990); M.P. Nightingale and H.W. J.Blote, Phys. Rev. B 33, 659 (1986).

[7] Zhong-Yi Lu, Zhao-Bin Su,and Lu Yu, Phys. Rev. Lett. 74, 4297 (1995).

[8] J.F. DiTusa et al, Phys. Rev. Lett. 73,1857 (1994).

[9] S.R. White, Phys. Rev. B 48,10345 (1993).

[10] S.R. White and R.M. Noack, Phys. Rev. Lett. 68, 3487 (1992).

[11] S.R.White, Phys. Rev. Lett. 69, 2863 (1992); S.R.White and D.A.Huse, Phys. Rev. B 48, 3844 (1993).

[12] E. S. Sorenson and I. Affleck, preprint 1994.

[13] O.Golinelli et al, Phys. Rev. B 50, 3037 (1994).

[14] see,e.g.,C.Bresinski and M.R.Zaglia Extrapolation Methods(Elsevier,New York,1991).

[15] M.Kaburagi et al, J. of Phys. Soc. of Japan 62,1848 (1993). 


\section{FIGURES}

FIG. 1. Impurity energy level vs impurity dev $=\left(J-J^{\prime}\right) / J$, when $0<J^{\prime}<J$. The chain is periodic, and the dashed line indicates the Haldane gap.

FIG. 2. Impurity energy level vs impurity dev $=\left(J^{\prime}-J\right) / J^{\prime}$, when $J^{\prime}>J$. The chain is periodic, and the dashed line indicates the Haldane gap.

FIG. 3. $<S_{z i}>$ of the impurity state as function of chain index $i$ when $J^{\prime}<J$. The star is for the case of $d e v=1$, while the triangle is for dev $=0.6$, and the square for dev $=0$ $\left(\right.$ dev $\left.=\left(J-J^{\prime}\right) / J\right)$. The impurity bond is placed between site 15 and 16.

FIG. 4. $<S_{z i}>$ of the impurity state as funtion of chain index $i$ when $J^{\prime}>J$. The star is for the case of $d e v=0.999$, while the triangle is for dev $=0.7$, and the square for dev $=0$ $\left(\right.$ dev $\left.=\left(J^{\prime}-J\right) / J^{\prime}\right)$. The impurity bond is placed between site 15 and 16 . 


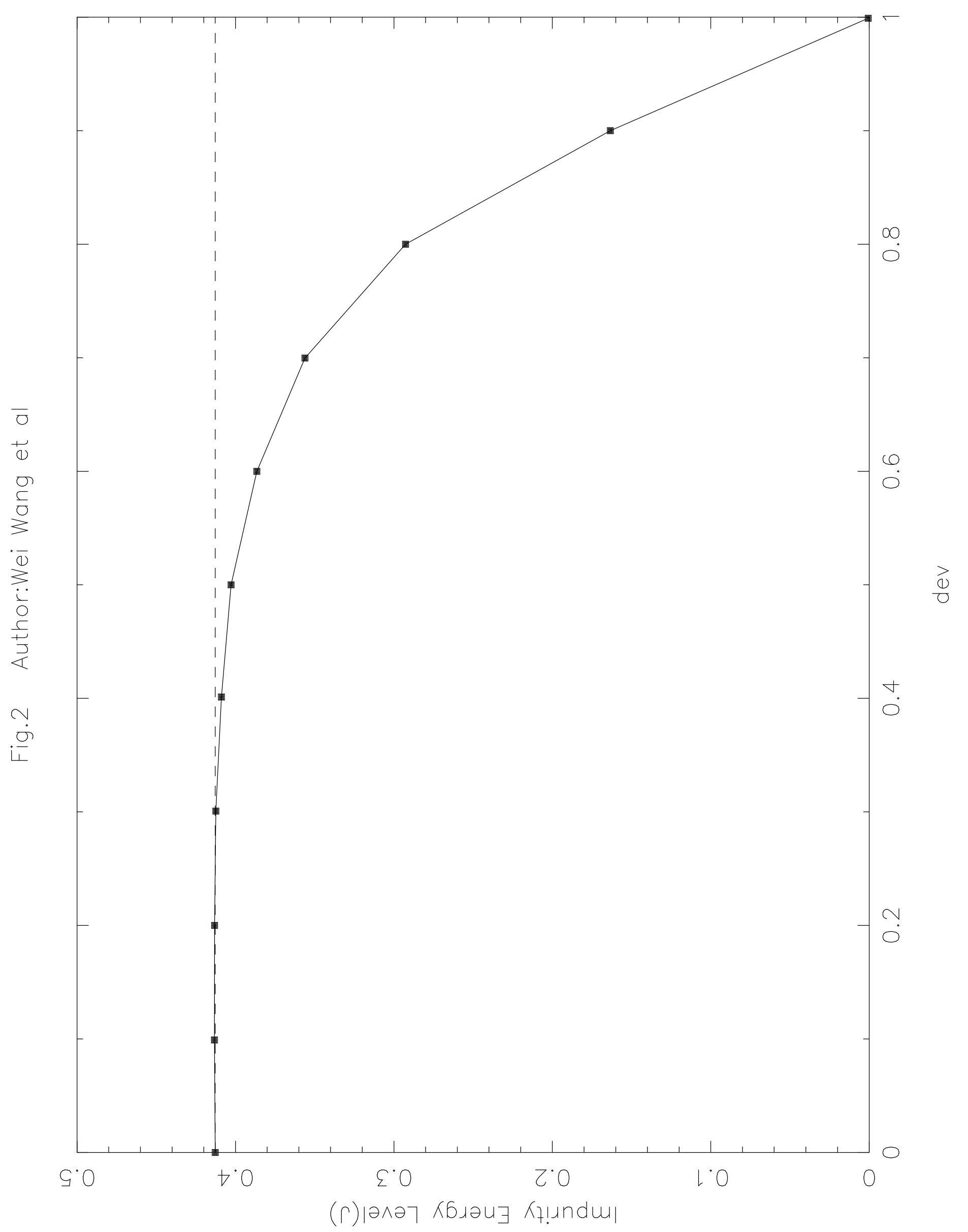




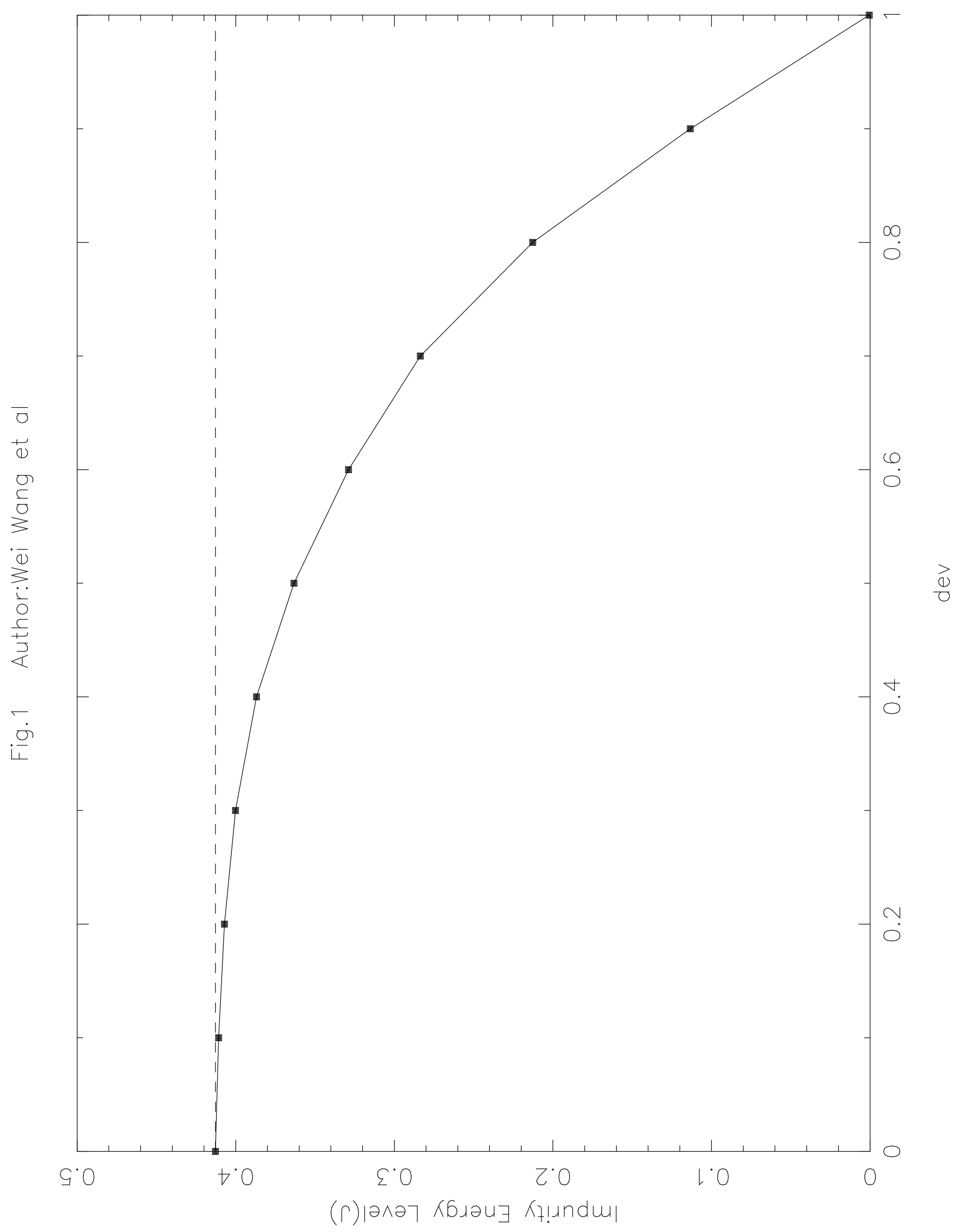




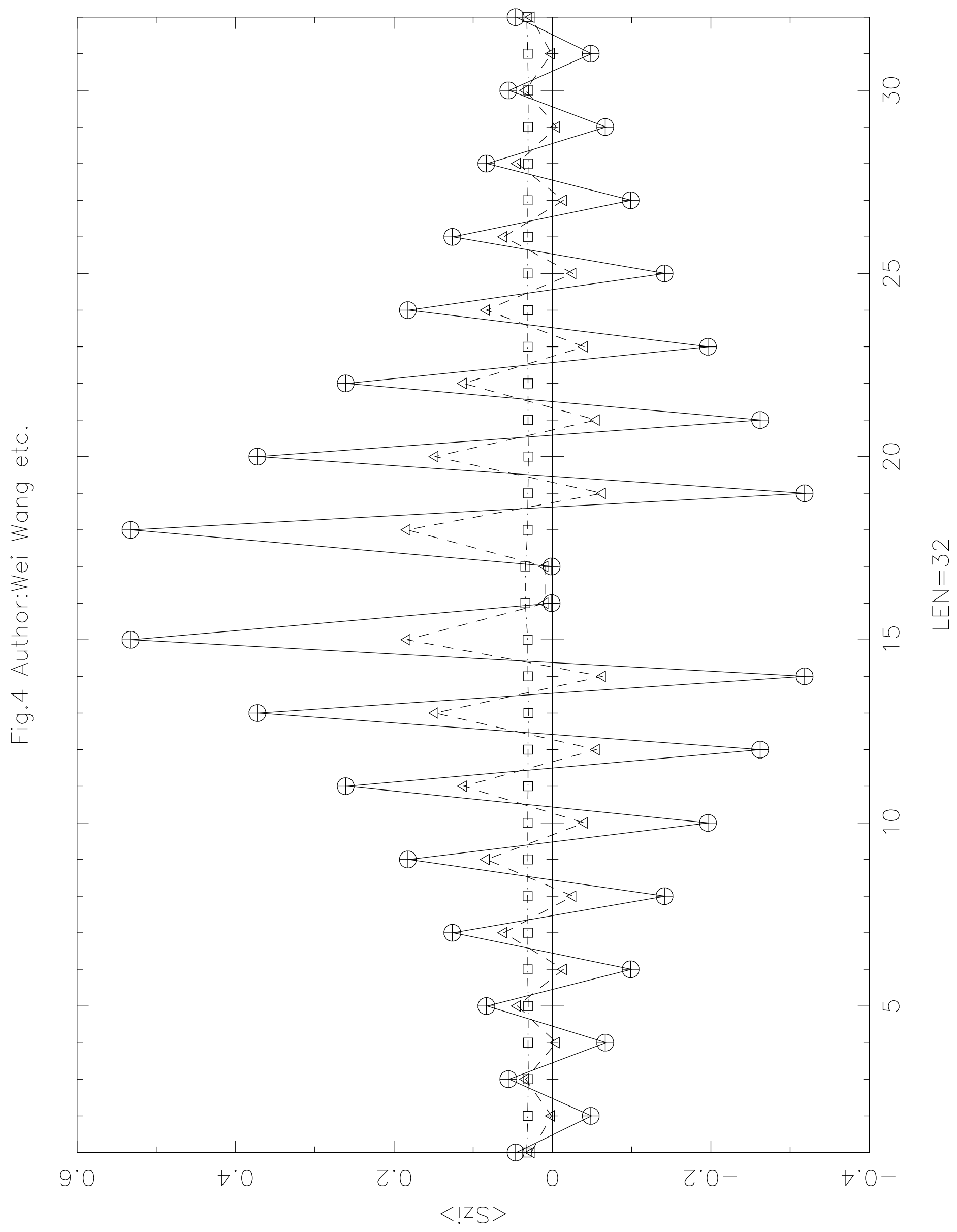




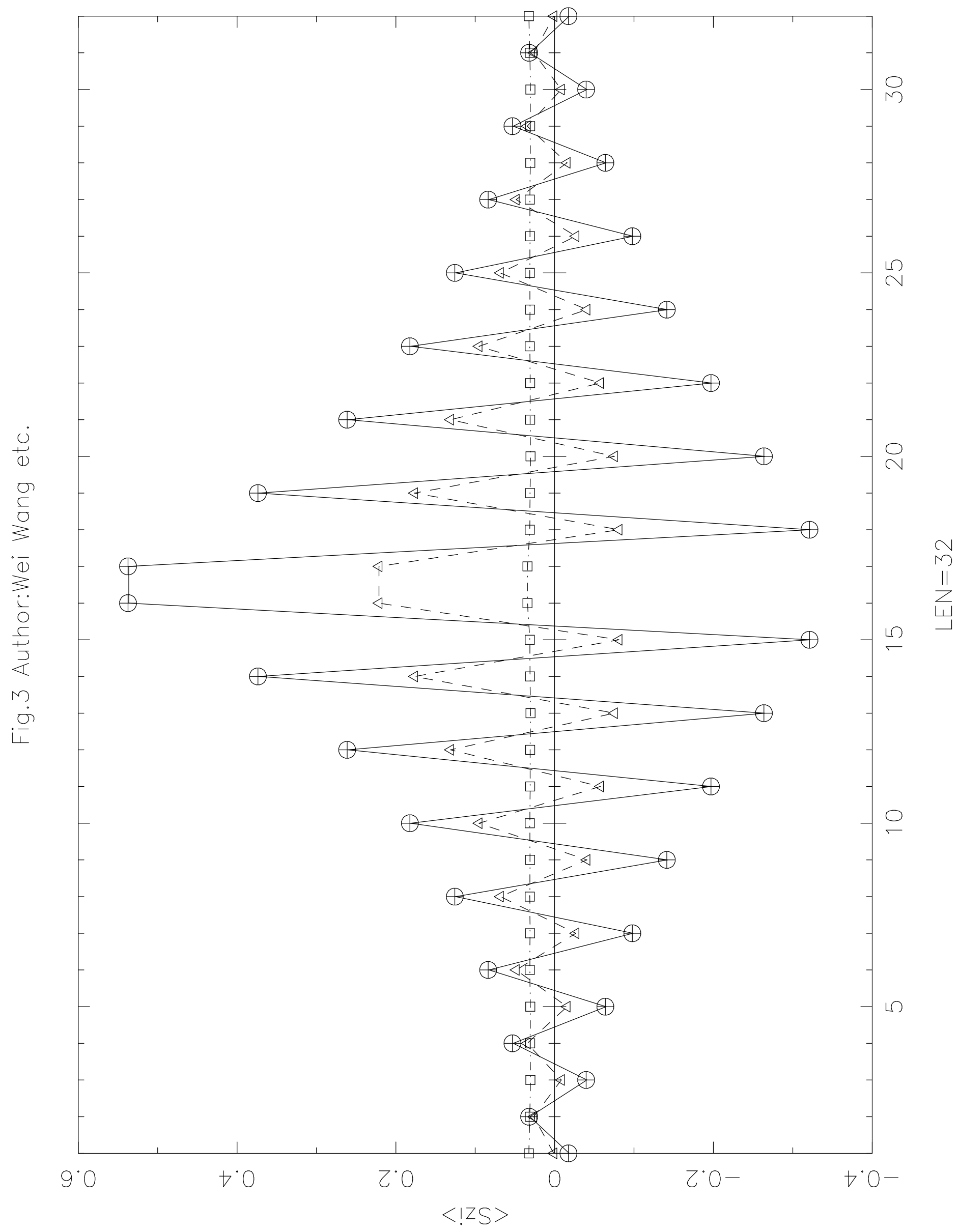

